\title{
Predicting the fungal CUG codon translation with Bagheera
}

\author{
Stefanie Mühlhausen and Martin Kollmar ${ }^{*}$
}

\begin{abstract}
Background: Many eukaryotes have been shown to use alternative schemes to the universal genetic code. While most Saccharomycetes, including Saccharomyces cerevisiae, use the standard genetic code translating the CUG codon as leucine, some yeasts, including many but not all of the "Candida", translate the same codon as serine. It has been proposed that the change in codon identity was accomplished by an almost complete loss of the original CUG codons, making the CUG positions within the extant species highly discriminative for the one or other translation scheme.

Results: In order to improve the prediction of genes in yeast species by providing the correct CUG decoding scheme we implemented a web server, called Bagheera, that allows determining the most probable CUG codon translation for a given transcriptome or genome assembly based on extensive reference data. As reference data we use 2071 manually assembled and annotated sequences from 38 cytoskeletal and motor proteins belonging to 79 yeast species. The web service includes a pipeline, which starts with predicting and aligning homologous genes to the reference data. CUG codon positions within the predicted genes are analysed with respect to amino acid

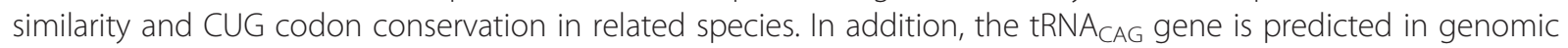
data and compared to known leu-tRNA $A_{C A G}$ and ser-tRNA ${ }_{C A G}$ genes. Bagheera can also be used to evaluate any mRNA and protein sequence data with the codon usage of the respective species. The usage of the system has been demonstrated by analysing six genomes not included in the reference data.

Conclusions: Gene prediction and consecutive comparison with reference data from other Saccharomycetes are sufficient to predict the most probable decoding scheme for CUG codons. This approach has been implemented into Bagheera (http://www.motorprotein.de/bagheera).
\end{abstract}

\section{Background}

For a long time it is known that many organisms show alterations to the universal genetic code $[1,2]$. These codon reassignments could have happened under strong AT or GC pressure, which might lead to the complete disappearance of the reassigned codon followed by a tRNA with a different amino acid identity taking over the decoding of the respective codon during its reappearance ("codon capture" theory [3]). In a mutually exclusive scenario, the codon is in a transitional state, in which it is decoded ambiguously by two tRNAs ("ambiguous intermediate" theory [4]). An example for the latter scenario is the reassignment of the CUG codon from leucine to serine in

\footnotetext{
*Correspondence: mako@nmr.mpibpc.mpg.de

Group Systems Biology of Motor Proteins, Department of NMR-based Structural Biology, Max-Planck-Institute for Biophysical Chemistry, Göttingen, Germany
}

Candida yeasts [5-7], which cannot be accomplished by a single mutation in the anticodon of a serine tRNA. Indeed, Candida species contain a single tRNA with a CAG anticodon (Ser-tRNA ${ }_{\text {CAG }}$ ) [8].

The general time line of the switch in using the leucine CUG codon for serine in fungi has already been investigated. Shortly, the unusual Ser-tRNA $\mathrm{CAG}_{\text {appeared about }}$ 270 million years (Ma) ago. However, the genera Candida (CUG codes for serine) and Saccharomyces (CUG codes for leucine) separated from each other about $180 \mathrm{Ma}$ ago implying that the codon ambiguity remained for about $100 \mathrm{Ma}$ in the ancestors of the yeasts [8,9]. The ancestor of the Saccharomyces lost the mutant Ser-tRNA $\mathrm{CAG}_{\text {and }}$

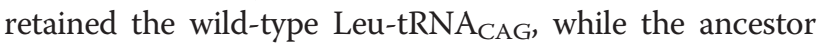
of the Candida lost the Leu-tRNA $\mathrm{CAG}_{\mathrm{C}}$ and maintained the mutant Ser-tRNA $\mathrm{CAG}_{\mathrm{G}}$ changing the identity of the CUG codon from leucine to serine. A whole genome 
comparison showed that only a minor fraction of the CUG codons present in Candida albicans have equivalent CUGs in Saccharomyces cerevisiae implying that almost all original CUG codons disappeared in C.albicans [9].

However, the decoding cannot be derived unambiguously from the species names (e.g. "Candida" species exist all over the yeast tree [10-13]). In several taxonomically broad protein family analyses [14-16] we have observed that CUG positions are conserved within many of these sequences, that many mapped to structurally conserved residues and can thus often unambiguously assigned to either leucine (large hydrophobic residue, at alignment positions highly enriched in hydrophobic residues) or serine (small polar residue). These observations also suggest that the data can be used as reference for the assignment of the CUG codon translation to further sequenced yeast species in the future.

Here, we provide a tool with which it can fast and easily be determined whether a yeast species uses the Standard Codon Usage or the Alternative Yeast Codon Usage (AYCU). The tool is suitable for both data from whole genome projects and transcriptome analyses. The tool is also thought to provide a reference page for species using the AYCU. In addition, the tool allows easy examination of the correct decoding of existing annotated genes by translating mRNA using the Standard or Alternative Yeast Codon Usage and by verifying the translation of a given protein sequence via gene reconstruction in the respective species. The tool closes an important gap in yeast research because the NCBI taxonomy does not reflect the latest phylogeny, and the assignment of the genetic code at the NCBI webpages is wrong for many species. E.g. Lodderomyces elongisporus, Hyphopichia burtonii, Candida tenuis, and others are denoted as using the Standard Code instead of the AYCU, which is known for e.g. Lodderomyces [17] and C.tenuis [7] for many years.

\section{Implementation Technologies}

The system has been developed to run on Linux systems. The web application is implemented in the Ruby programming language (version 1.9.3; [18]) using the Ruby on Rails framework (version 3.2.12; [19]), which has the advantage of rapid and agile development while keeping the code well organized. The site makes extensive use of Ajax (Asynchronous JavaScript and XML) in order to present the user with a feature rich interface while minimizing the amount of transferred data. The alignments and phylogenetic trees are visualized with the Lucullus software, which is a plugin to PyBioMaps [20]. Performance is enhanced by parallelization of the prediction process. All technologies used are freely available and open source.

\section{Workflow}

The implementation of the CUG prediction workflow is shown in Figure 1A. The uploaded yeast genome or transcriptome assembly data is searched with representatives of 2071 proteins from 38 different protein families and classes using TBLASTN [21,22]. The protein reference dataset is updated from CyMoBase $[23,24]$ on a monthly basis. The genomic regions of the BLAST hits are extended by 500 nucleotides in both directions to obtain better and more complete $a b$ initio gene predictions. Subsequently, overlapping BLAST hits are combined because they presumably belong to the same gene. Genes within these extended genomic regions are predicted by AUGUSTUS-PPX $[25,26]$ with the options -genemodel=exactlyone to predict exactly one gene, -proteinprofile to integrate pre-calculated profiles for each protein family of the reference data, and -species_model to use species-specific parameters. Subsequently, the AUGUSTUS predictions are aligned to the reference multiple sequence alignments with MAFFT [27], or to the reference sequence used for the TBLASTN search with $\mathrm{C}++$ implementations of the Needleman-Wunsch [28], Gotoh [29], Smith-Waterman [30] or Longest Common Subsequence [31] algorithms, which are part of the SeqAn algorithm library [32]. CUG positions within the predicted genes are then compared to the reference data with respect to amino acid conservation and, if existing, to the translations of CUG codons at the same position in the reference data. Optionally, the user can compute the phylogenetic grouping of the yeast query data to the reference data. For this purpose, five or ten of the predicted sequences are chosen randomly, the corresponding alignments of the gene predictions and reference data are concatenated, poorly aligned positions are removed with Gblocks v.0.91b [33], and the phylogenetic tree is computed with FastTree [34]. In addition to this sequence-based prediction of the translation scheme, the identity of the $\mathrm{tRNA}_{\mathrm{CAG}}$ is predicted (Figure 1A). For this purpose, tRNAs and their secondary structures are predicted with tRNAscan-SE under a general (-G) or a eukaryote-specific tRNA model [35]. Pseudogene checking is disabled with option $-\mathrm{D}$ to speed up the search process. The predicted $t \mathrm{RNA}_{\mathrm{CAG}}$ is subsequently compared to reference data consisting of 51 leutRNA $_{\text {CAG }}$ genes, 22 ser-tRNA ${ }_{\text {CAG }}$ genes, and 34 tRNA genes with other anticodons. For visual inspection, the predicted tRNA $_{C A G}$ is aligned to the reference tRNA data with MAFFT.

The workflow for the verification of the CUG translation in a given protein sequence is shown in Figure 1B. Shortly, a gene reconstruction of the query protein in the selected species is performed with WebScipio [36]. Optionally, the gene reconstruction can be performed with less stringent parameters, which include relaxed values for the parameters -minimal identity, --maximal 


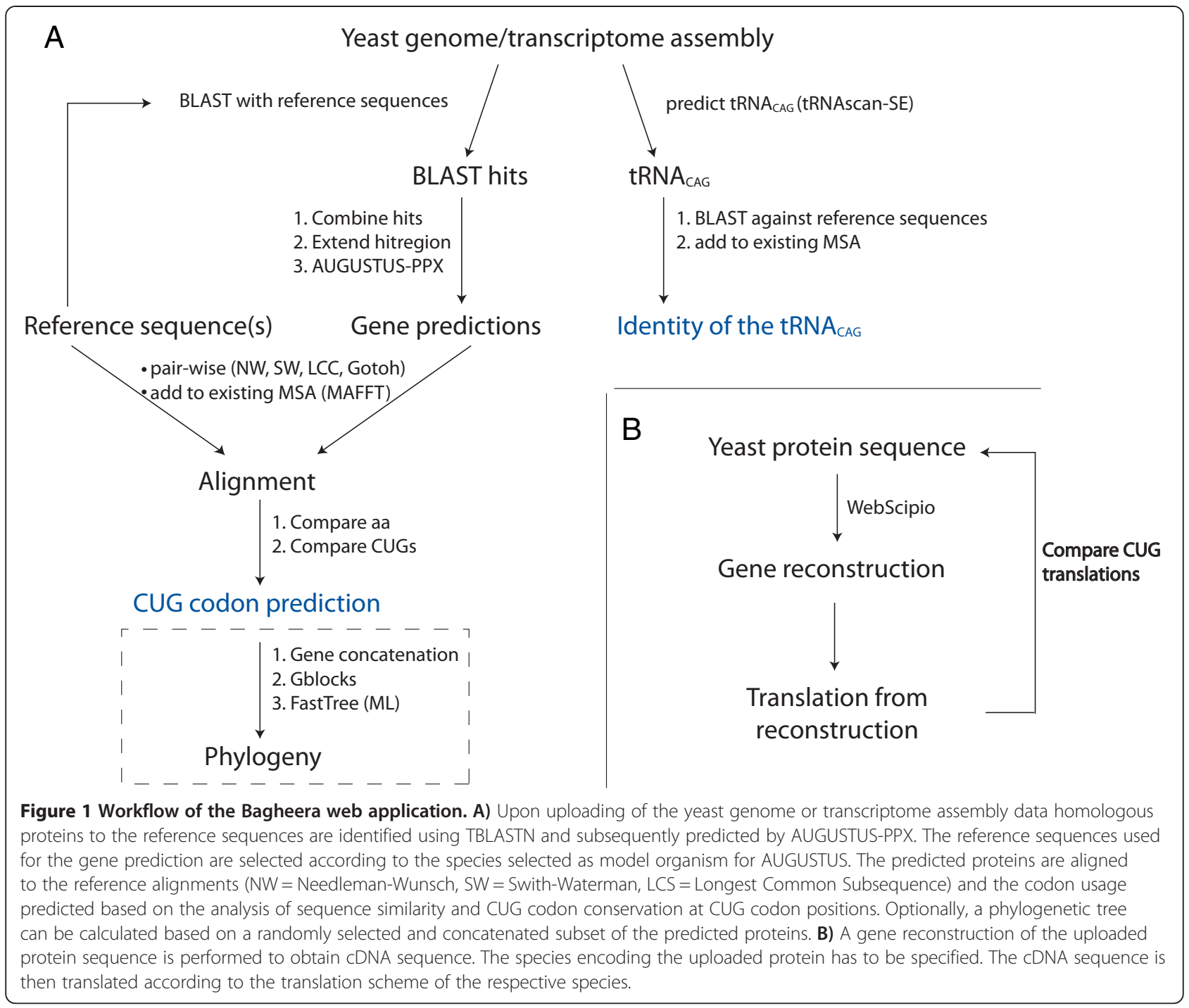

mismatches and -minimal score to allow prediction of less similar genes. This option is very useful if the respective query gene has not been derived from one of the reference species but a closely related one. The coding DNA obtained from the gene reconstruction is retranslated into protein sequence using the translation scheme of the selected species, which is already implemented in WebScipio.

For the translation of a provided mRNA into protein, the mRNA is first split into codons, which are then translated using the specified translation scheme. Extra nucleotides are ignored.

\section{Identification and annotation of the reference data}

Fungal actin and actin-related proteins, dynactin proteins, myosins, kinesins, dynein heavy chains, tubulins, actincapping proteins CapZ, coronins and WASP homologs have been extracted from previously published datasets
[14-16,37]. The sequences were updated based on newer genome assemblies if necessary. The reference data for the other proteins and the species not included in the published datasets have essentially been obtained as described in [14]. Shortly, the corresponding genes have been identified in TBLASTN searches starting with the respective protein sequence of homologs of Saccharomyces cerevisiae. The respective genomic regions were submitted to AUGUSTUS [25] to obtain gene predictions. However, feature sets are only available for a few species of the Saccharomycetes clade. Therefore, all hits were subsequently manually analysed at the genomic DNA level. When necessary, gene predictions were corrected by comparison with the homologs already included in the multiple sequence alignments.

Reference tRNA ${ }_{C A G}$ genes were predicted with tRNAscan$\mathrm{SE}$ in 45 yeast species. Intron regions were removed and tRNAs aligned manually. 
All sequence related data (protein names, corresponding species, sequences, and gene structure reconstructions) and references to genome sequencing centres are available at CyMoBase (http://www.cymobase.org, [23]). A list of the reference species and their abbreviations as used in the alignments and trees, as well as anamorph and alternative names can be accessed through the web server and as Additional file 1. Additional file 1 also includes references to published genomes, and detailed information and acknowledgments of the respective sequencing centres. All gene structures for the reference dataset have been reconstructed with Scipio/WebScipio $[36,38]$.

\section{Results and discussion}

The first step in gene annotation is gene prediction, which can be done in a genome-wide scan or for single genes. Many gene prediction programs allow using different codon translation tables, but this option is not available in most of the gene prediction web interfaces. Even then, it would require the user to know a priori, whether the target organism belongs to the species not using the standard codon table. Especially the yeasts are confusing, as the "Candida" species are well known to use the AYCU in contrast to Saccharomyces cerevisiae. But this has only been shown for a few Candida species, including some of the most pathogenic, and many yeast species are called Candida although there is no monophyletic "Candida clade". Codon decoding schemes cannot unambiguously be derived from single-gene studies because the respective gene might not contain the codon in question at all, or the respective amino acids are not at meaningful positions. Meaningful positions would be those that are strongly conserved in evolution and therefore in the core of the proteins or at conserved binding interfaces at the surface. In the course of our continuous efforts in identifying and annotating cytoskeletal and motor proteins [14-16,37] we have already assembled and annotated 2071 sequences from 18 protein families in 79 yeast species. Some of the data has already been used to evaluate the CUG encoding in 60 completely sequenced yeasts (Mühlhausen and Kollmar, unpublished data). Here, these data are used as reference dataset in a pipeline for the prediction of the CUG codon translation, which can be accessed by users through a web interface.

\section{The reference data}

Currently, CyMoBase [23,24], a database for manually assembled and annotated cytoskeletal and motor proteins, contains 26 protein families with annotated proteins in 79 yeast species. All sub-families of these protein families, like for example the $\alpha$-, $\beta$-, and $\gamma$-tubulins, already existed in the last common ancestor of the opisthokonts or even the eukaryotes and are therefore treated as independent proteins. Not all protein families in CyMoBase have been analysed at the same depth, e.g. only two dynein lightintermediate chain proteins are available yet. Also, some sub-families like the class- 17 myosins or the class- 4 kinesins are only present in early diverging yeast species and not in e.g. Candida albicans or Saccharomyces cerevisiae. These proteins do not provide the necessary statistical basis and taxonomic sampling for a CUG prediction and were not included in the Bagheera reference data. Bagheera's reference data thus consists of 18 protein families (38 independent proteins) comprising 2071 sequences. These data will increase in the future in the course of our continuous efforts in annotating cytoskeletal and motor proteins. Most of the reference proteins are considerably longer then the average yeast proteins like for example the myosins (1100 to 2400 amino acids) and the dynein heavy chain proteins (about 4000 amino acids), and the reference data therefore comprises significantly more data than the sole numbers of proteins and sequences might implicate (Additional files 1 and 2).

\section{The web interface}

Great attention has been paid to a versatile yet easy to use web interface (Figure 2). We think that accessibility and high quality representation is key to a productive usage of the system. Bagheera offers possibilities to analyse largescale, whole genome and transcriptome assembly data, and to determine the correct CUG translation for any single cDNA or protein sequence.

Regarding the prediction of the most probable CUG translation in large-scale data, Bagheera does the following: i) The user provides genomic data, e.g. a genome assembly, transcriptome assembly or long-read EST data, or data from single to multiple gene analyses. ii) The tool predicts cytoskeletal and motor proteins and aligns the predicted sequences to the respective protein families. iii) The respective positions of the CUG codons of the predicted sequences are compared to the reference data. iv) The tool predicts RNA $_{\text {CAG }}$ and performs a sequence similarity search and sequence alignment with reference leu-tRNA $\mathrm{CAG}_{\mathrm{C}}$ and ser-tRNA $\mathrm{CAG}_{\mathrm{G}}$ genes.

The verification of the translation of a single sequence depends on the provided sequence. The translation of a given cDNA sequence is done as follows: i) The user provides an mRNA sequence and specifies the translation scheme. ii) The tool translates the mRNA into protein. For any given protein sequence the workflow is: i) The user provides a protein sequence and specifies the corresponding species. ii) The tool performs a gene reconstruction for the protein with WebScipio and retranslates the coding regions using the corresponding CUG translation scheme as provided by WebScipio. iii) CUG translations in the user-provided protein are compared to translations in the re-translated. 


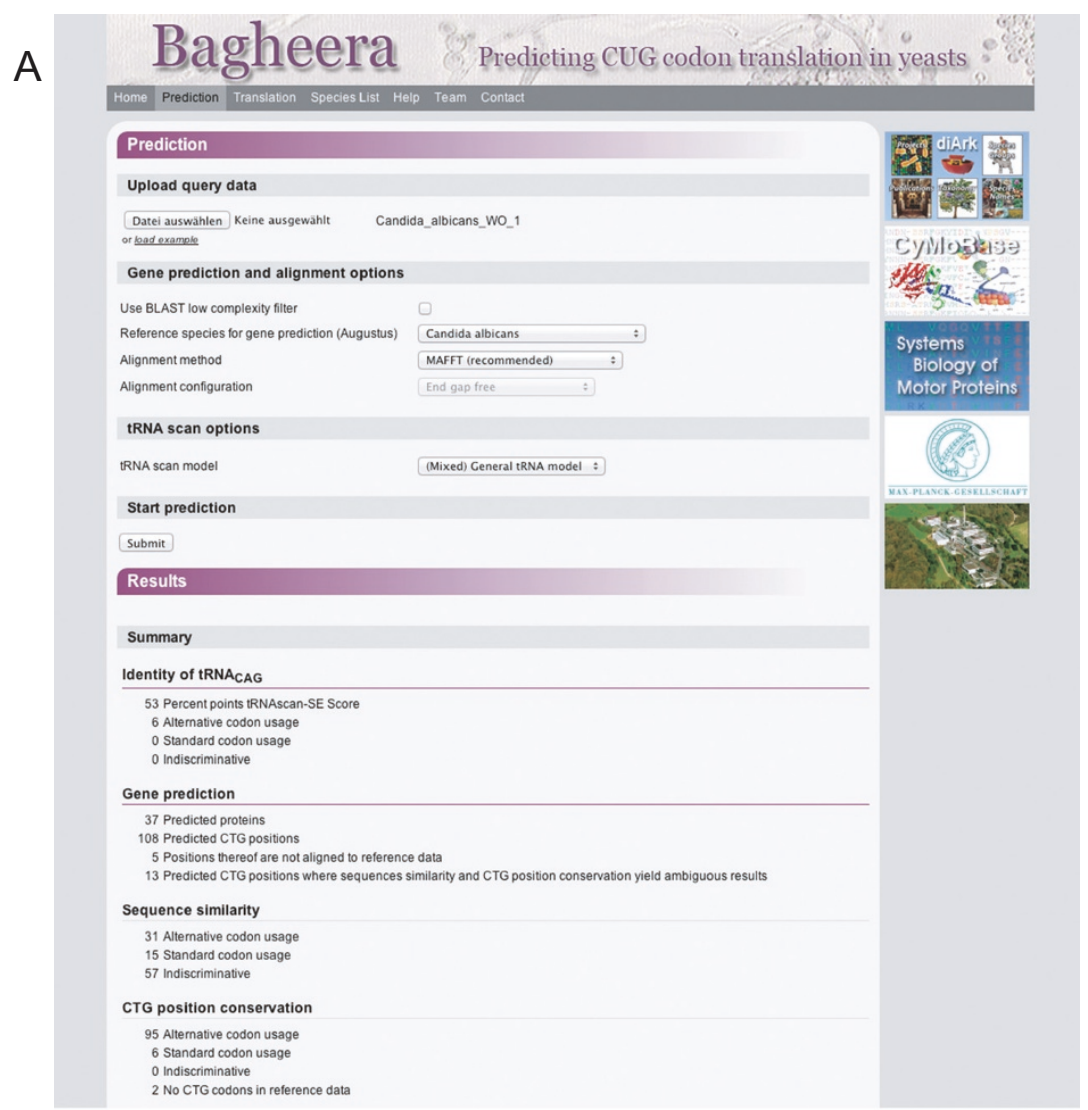

B

Actin related protein Class 4 (Hit 1/15)

\section{quence similarity}

CTG Position Distribution of amino acids in reference data Number of amino acids

1 CTG position suggests alternative codon usage.

CTG position conservation

CTG Position CTG usage in reference data Number of CTG codons

1 CTG position suggests alternative codon usage.

71 sequences in reference alignment

Show predicted sequence $\pi$

CTG
position

MATASTTTVYGGDEINAIVLDPGSYTTRIGYAGDDFPKVITSSYYGQVENDKKKIFGESINVPRANYDIKPILKESIIVD

WDAAIEQYQYYFDQQLKVVGPEQPILITEPIWTETSYRQQLVETFFENFEFSGIYLAKSPTCVSPQQGRSNCLVVDLGHD

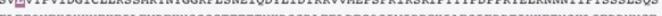

ISTTQANEYRPLKRVRKNDDEEEEDSNOSTPKPEVNIRGISQLISHTLSNIDIDLRASLANNI IVTGGVSLISQLTERLY

LELSNNNPGLKIRLHAVGNSTERINQAWIGGKY

Predicted sequence length 433 amino acids

Reterence species Candida albicans SC 5314

Show alignment $\pi$

Index of CTG position[s] in the alignment

Index of CTG position/s in the alignment
CTG Position in sequence CTG Position in alignment
163.291

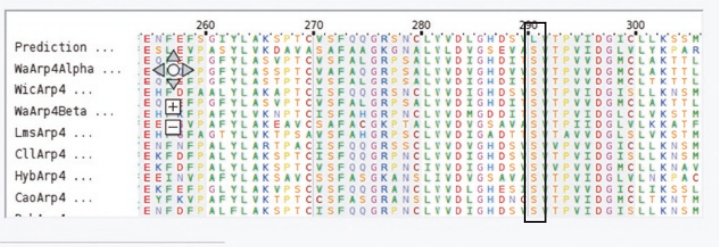

Use next BLAST hits for gene prediction $v$

Figure 2 (See legend on next page.) 
(See figure on previous page.)

Figure 2 Screenshot of the web interface. The web interface is divided into three main parts: data upload and options section, results section, and phylogenetic tree section (not shown). A) Example data were uploaded and processed with default parameters. B) The results section is split into a summary and a section listing each reference protein and a detailed analysis of each predicted protein down to single CUG codons. For every reference protein, the predicted gene and, if applicable, the respective CUG positions are shown. For every predicted CUG position, which could be mapped onto the reference data, the amino acid composition and CUG codon usage at the respective positions in the reference data are listed. The predicted actin related protein class 4 (Arp4) contains one CUG at position 163. This position corresponds to alignment position 291 in the reference alignment. It is here indicated by a black box. All CUG codons are noted as leucine in the predicted sequence, regardless the suggested codon usage.

Care has to be taken when using transcriptome assembly or long-read EST data as input. Transcriptome data do not represent all coding regions of a species, which might lead to wrong assignment of predicted proteins to the reference data. For example, actin and the actinrelated proteins are closely related, or the $\alpha-, \beta-$, and $\gamma^{-}$ tubulins, or the members of the other multi-gene protein families. In the case that only actin genes are present in the transcriptome data these would be identified as closest homologs and aligned to all of the actin-related proteins of the reference data. While key residues for folding and ATP-binding are of course conserved between actin and all actin-related proteins, residues in loop regions are less or not at all conserved. Within the same sub-family these regions could also contain valuable information, because loops might be conserved within the entire sub-family. By aligning proteins from different sub-families this information might at best be lost or in the worst case even lead to contradicting results.

In the first step of the prediction pipeline, homologs to the proteins of the reference data need to be identified. Here, one gene is predicted for every protein family present in the reference data using TBLASTN [21] and AUGUSTUS-PPX $[25,26]$. We choose BLAST as search algorithm because it is very fast and not as restrictive in terms of sequence homology as for example BLAT [39]. To optimize the search and subsequent gene prediction the user can select one of the AUGUSTUS feature sets, which contain species-optimized parameters and are available for a number of yeast species. The reference proteins used in the BLAST search are taken from the species, which had been selected for the AUGUSTUS prediction. In most cases, the BLAST hits do not cover the entire genes but miss the $\mathrm{N}$ - and $\mathrm{C}$-termini, and low complexity regions. In the latter case and in the case that genes are split into several exons, the search results in several BLAST hits belonging to the same gene. These partial hits are combined and extended in the $5^{\prime}$ and 3 ' direction because AUGUSTUS gene predictions are significantly better when intergenic regions are included in the genomic regions.

\section{Prediction of the CUG codon translation}

In the second step, the most probable codon usage in the predicted sequences is determined. To this end, the predicted sequences are aligned to the multiple sequence alignments of the respective protein families or, optionally, only to the reference sequence, which has been used in the BLAST search. We choose MAFFT [27] as default alignment method, because it allows adding a new sequence to an already existing multiple sequence alignment. Based on these alignments two features are analysed: CUG position and amino acid conservation. The reference data contains 8244 known CUG codons (Figure 3, Additional file 3), and it is determined whether CUG codons in the query data match CUG codons in the reference data. In addition, the amino acid compositions at alignment positions, where the query data contain CUGs, are determined. The usage of reference genes and their encoding of CUG codons are restricted to completely assembled genes, while the reference amino acid composition is also calculated on basis of incompletely assembled genes. Based on these data, the most probable codon usage for every CUG codon is predicted. Here, the encoding of CUG codons in the reference data as leucine and the presence of hydrophobic amino acids (leucine, isoleucine, valine, methionine, phenylalanine) at the respective alignment position are taken as indicator for the standard codon usage while CUG codons encoding serine and a preference for the polar and small amino acids serine, threonine, cysteine, and alanine are taken as indicator for the yeast alternative codon usage. However, the predictive power of the CUG positions is not equal. Positions important for protein folding, which are usually in the core of the proteins, and those important for protein interactions and ligand binding have a higher significance as those in loop regions at the protein surface. Therefore, we provide separate evaluations for the CUG codon position conservation and the amino acid similarity at CUG codon positions in the alignment. By analysing the genomes of yeast species with known CUG translation schemes, Yarrowia lipolytica, Candida glabrata and Saccharomyces cerevisiae using the Standard Code, and Candida albicans, Debaryomyces hansenii and Lodderomyces elongisporus using the AYCU, we found out that a simple majority rule is sufficient to predict the CUG codon translation scheme. Therefore, whatever the majority of the reference data is at a given CUG codon position, this will count for either the Standard or the AYCU. As a third option, the result is termed ambiguous or indiscriminative 


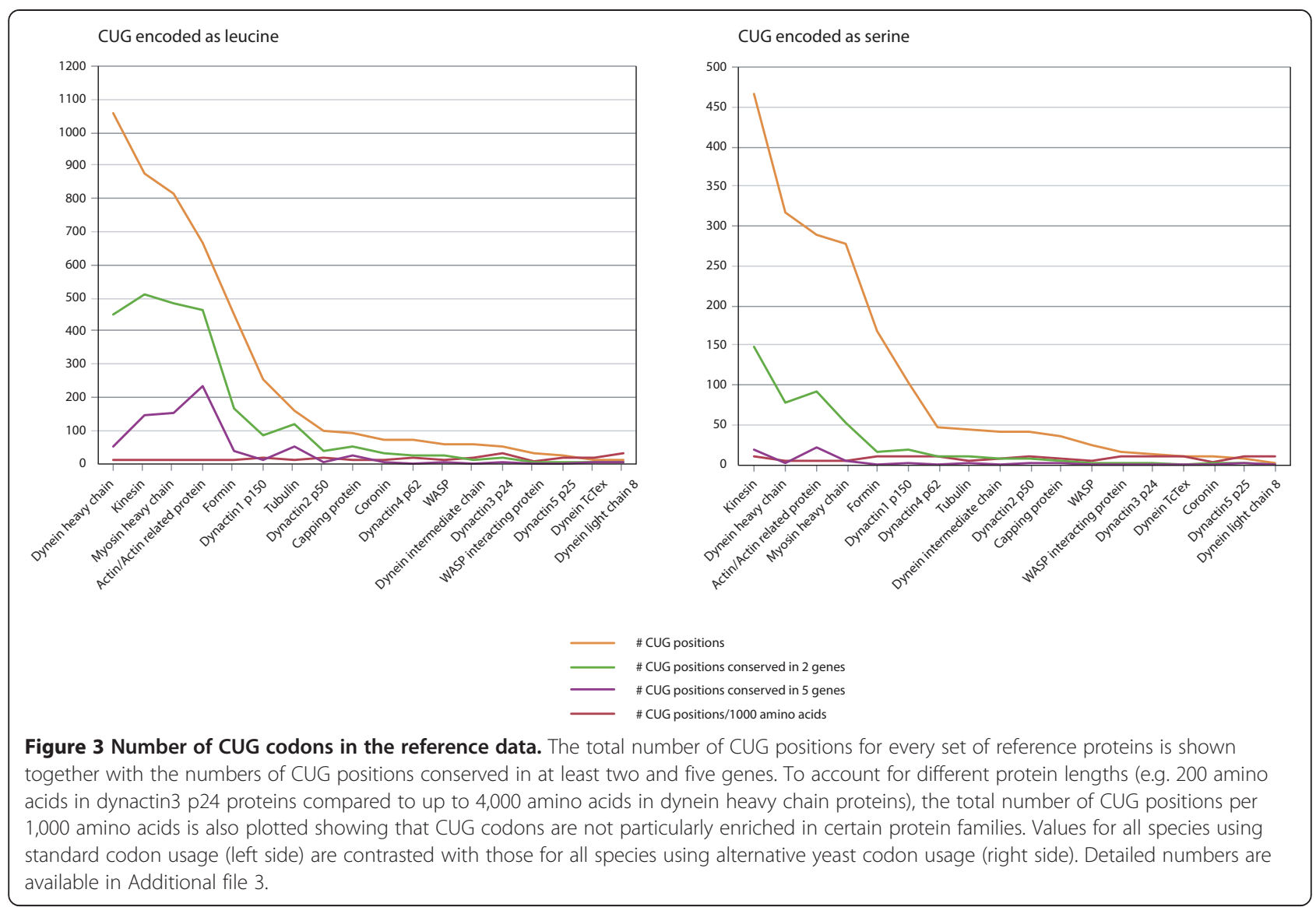

if the majority of the residues at CUG codon positions do not belong to either large hydrophobic residues ( $=>$ Standard Codon usage) or small polar residues $(=>$ AYCU). The proposed CUG codon translation for the query sequence (genome or transcriptome assembly) will be given for every single reference protein and in summary for all reference data.

\section{Prediction of the tRNA CAG $_{\text {}}$}

Independent support for the proposed translation scheme is provided by tRNA prediction, which is performed with tRNAscan-SE. Subsequently, a BLAST search against reference leu-tRNA ${ }_{C A G}$ and ser-tRNA $A_{C A G}$ genes indicates the most probable identity of the $\mathrm{tRNA}_{\mathrm{CAG}}$ in the query data. In addition, the predicted $\mathrm{tRNA}_{\mathrm{CAG}}$ is aligned with the reference data for visual inspection.

\section{Translation check}

Any given protein sequence can be checked for correct translation of CUG codons. This is an important option for all users who obtained protein sequences from databases, which did not resolve the codon translation yet. For example, at species genome project homepages gene annotations are often provided with non-uniform translations. All CUG codons are highlighted, differences between the correct and the given translation are indicated. If the given translation is partially incorrect, the correctly translated protein sequence can be downloaded in fasta-format.

\section{Case study}

As example for the usability of Bagheera we choose Candida bracarensis, Candida castellii, Candida maltosa, Candida nivariensis, Nakaseomyces bacillisporus, and Nakaseomyces delphensis because these are not yet included in CyMoBase's reference data (Table 1). The genome assemblies were obtained from NCBI and uploaded into Bagheera. At NCBI, C.bracarensis and C.nivariensis are still grouped to the Candida branch (mitosporic Saccharomycetales) although a recent whole genome analysis showed their close homology to Candida glabrata, which belongs to the Saccharomycetaceae [40]. Therefore, we selected the "Candida albicans" feature set for C.maltosa and the "Saccharomyces cerevisiae" feature set for the other species for the gene prediction with AUGUSTUS. In all six genomes, homologs of almost all of the 38 reference proteins from Candida albicans and Saccharomyces cerevisiae were predicted (Table 1) supporting the 
Table 1 Details of the CUG codon prediction in the genomes of six yeast species

\begin{tabular}{|c|c|c|c|c|c|c|c|c|c|}
\hline \multirow[t]{2}{*}{ Species } & \multirow[t]{2}{*}{$\begin{array}{l}\text { Predicted } \\
\text { proteins }\end{array}$} & \multirow[t]{2}{*}{$\begin{array}{l}\text { Proteins } \\
\text { with CUG }\end{array}$} & \multirow[t]{2}{*}{$\begin{array}{l}\text { CUG } \\
\text { codons }\end{array}$} & \multicolumn{2}{|c|}{$\begin{array}{l}\text { CUG position } \\
\text { conservation }\end{array}$} & \multicolumn{2}{|l|}{$\begin{array}{l}\text { Sequence } \\
\text { similarity }\end{array}$} & \multirow[t]{2}{*}{ tRNA $_{C A G}$} & \multirow[t]{2}{*}{$\begin{array}{l}\text { Accession } \\
\text { number }\end{array}$} \\
\hline & & & & $\begin{array}{l}\text { Std codon } \\
\text { usage }\end{array}$ & AYCU & $\begin{array}{l}\text { Std codon } \\
\text { usage }\end{array}$ & AYCU & & \\
\hline Candida bracarensis CBS 10154 & 37 & 31 & 174 & 140 & 2 & 145 & 8 & n.d. & CAPU00000000 \\
\hline Candida castellii CBS 4332 & 35 & 30 & 185 & 142 & 2 & 138 & 11 & n.d. & CAPW00000000 \\
\hline Candida maltosa Xu316 & 34 & 20 & 71 & 4 & 35 & 18 & 14 & ser-tRNA ${ }_{C A G}$ & AOGT00000000 \\
\hline Candida nivariensis CBS 9983 & 34 & 30 & 182 & 131 & 2 & 150 & 7 & n.d. & CAPV00000000 \\
\hline Nakaseomyces bacillisporus CBS 7720 & 33 & 25 & 122 & 89 & 3 & 100 & 2 & n.d & CAPX00000000 \\
\hline Nakaseomyces delphensis CBS 2170 & 34 & 31 & 194 & 148 & 4 & 159 & 3 & n.d. & САРТ00000000 \\
\hline
\end{tabular}

completeness of the genome assemblies and similarity between the species. For C.maltosa 71 CUG codons were identified in 20 proteins, which is in agreement with the average number of CUG codons in the CTG clade (Mühlhausen and Kollmar, unpublished data). Accordingly, the genes of C.bracarensis, C.castellii, C.nivariensis, N.bacillisporus, and N.delphensis contain slightly more CUG codons (122 to 194 codons). In all five Saccharomycetaceae, about $75 \%$ of the CUG positions are conserved within the reference data. In C.maltosa about 50\% of the CUG positions are conserved. Together, these data propose the AYCU for C.maltosa and the standard translation table for C.bracarensis, C.castellii, C.nivariensis, N.bacillisporus, and N.delphensis. AYCU for C.maltosa has already been shown [41].

\section{Limits of Bagheera}

Possible limits of the tool might be that the query genes do not contain CUG codons and that the database contains only 18 protein families with 38 independent proteins. However, the proteins of most families of the reference data, e.g. myosins and dyneins, are very long and every of these proteins contains at least a few CUG codons (Figure 3). A whole genome sequence analyse will therefore always provide enough data for unambiguous assignment of the codon usage. Actins and tubulins also belong to the most widely used proteins for species phylogenies (e.g. recent analyses: [42-45]) and because of their high abundance in the cell it is highly likely that they are included in transcriptome assembly data and small-scale analyses. Although the presence of a leu-tRNA ${ }_{C A G}$ or sertRNA $_{\text {CAG }}$ gene is a very strong indication for the Standard or AYCU, these genes are often not present in the genomes (e.g. in Saccharomyces cerevisiae) or might contain extremely long introns of more than 250 nucleotides hindering their identification and prediction.

\section{Conclusions}

With this software we demonstrated that the most probable codon translation scheme for a given yeast genome can be determined by predicting motor and cytoskeletal proteins and comparing them to reference data. In total, 2071 sequences from 38 proteins belonging to 79 yeast species were included in the reference data providing a two-fold basis for the prediction of the most probable translation scheme: the amino acid composition at CUG positions and the conservation of CUG positions. The presence of hydrophobic amino acids in the reference data suggests the translation of the predicted CUG codons as leucine, while polar and small amino acids suggest their translation as serine. In addition, matching of CUG codons in the predicted genes with CUG codons in the reference data provides further support for the standard or alternative yeast codon usage. This information was implemented into a CUG codon prediction pipeline accessible via a web server called Bagheera. The predictive power of this implementation was demonstrated by a case study of the genomes of six Saccharomycetes species. In addition, the webserver offers the possibility to verify the translation of the CUG codons in any given protein sequence. Moreover, the webserver can be used as reference for the translation scheme used by individual yeast species.

\section{Availability and requirements}

Project name: Bagheera - Predicting CUG codon translation in yeasts

Project home page: http://www.motorprotein.de/bagheera Operating system: Platform independent

Programming language: Ruby

Other requirements: The current version of Bagheera has extensively been tested with Firefox version 15 or higher with JavaScript enabled, but should run on all modern browsers.

Licence: The source code for the web application and a command line tool can be obtained upon request and used under a Creative Commons License.

Any restrictions to use by non-academics: No. 


\section{Additional files}

Additional file 1: List of reference species. This file contains a table with detailed information about the species, including teleomorph, anamorph and alternative scientific names, the species abbreviations as used throughout the web server, credits for the sequencing centres, and references to published genome analyses. In addition, the number of genes obtained from CyMoBase is listed.

\section{Additional file 2: CUG codon positions and amino acid composition} of the reference data. This table lists the CUG codon positions and the amino acid composition at respective alignment positions for every reference protein. The data are separated into two different sheets. In the first sheet, the positions of CUG codons are listed for every reference gene. The amino acid composition at every CUG position is included in the second sheet.

Additional file 3: Number of CUG positions in the reference data and their conservation. The table lists the number of CUG positions within each set of reference proteins in total counts and normalized to the protein lengths. In addition, the numbers of conserved CUG positions in at least two and five genes are given. The data are separated by reference species encoding CUG as leucine (sheet 1) and serine (sheet 2). These values are plotted in Figure 3.

\section{Competing interests}

The authors declare that they have no competing interests.

\section{Authors' contributions}

SM and MK specified the requirements from a user's perspective, defined the rules for data handling, and collected all the reference data. SM did the technical design and the programming. MK and SM wrote the manuscript. Both authors read and approved the final manuscript.

\section{Acknowledgements}

We would like to thank Dr. Björn Hammesfahr for help with CyMoBase, Marcel Hellkamp for help with Lucullus, Fabian Meyer for help with Ajax, and Prof. Christian Griesinger for his continuous generous support. This project has been funded by grant KO 2251/13-1 of the Deutsche Forschungsgemeinschaft (DFG) and was partly supported by the Göttingen Graduate School of Neurosciences and Molecular Biosciences (DFG Grants GSC 226/1 and GSC 226/2)

Received: 9 November 2013 Accepted: 21 May 2014

Published: 29 May 2014

\section{References}

1. Jukes TH, Osawa S, Muto A, Lehman N: Evolution of anticodons: variations in the genetic code. Cold Spring Harb Symp Quant Biol 1987, 52:769-776.

2. Jukes TH, Osawa S: Evolutionary changes in the genetic code. Comp Biochem Physiol B 1993, 106:489-494.

3. Osawa S, Jukes TH: Codon reassignment (codon capture) in evolution. J Mol Evol 1989, 28:271-278.

4. Schultz DW, Yarus M: Transfer RNA mutation and the malleability of the genetic code. J Mol Biol 1994, 235:1377-1380.

5. Ohama T, Suzuki T, Mori M, Osawa S, Ueda T, Watanabe K, Nakase T: Non-universal decoding of the leucine codon CUG in several Candida species. Nucleic Acids Res 1993, 21:4039-4045.

6. Pesole G, Lotti M, Alberghina L, Saccone C: Evolutionary Origin of Nonuniversal Cug(ser) Codon in Some Candida Species as Inferred from a Molecular Phylogeny. Genetics 1995, 141:903-907.

7. Sugita T, Nakase T: Non-universal usage of the leucine CUG codon and the molecular phylogeny of the genus Candida. Syst Appl Microbio/ 1999, 22:79-86.

8. Yokogawa T, Suzuki T, Ueda T, Mori M, Ohama T, Kuchino Y, Yoshinari S, Motoki I, Nishikawa K, Osawa S: Serine tRNA complementary to the nonuniversal serine codon CUG in Candida cylindracea: evolutionary implications. Proc Natl Acad Sci USA 1992, 89:7408-7411.

9. Massey SE, Moura G, Beltrão P, Almeida R, Garey JR, Tuite MF, Santos MAS: Comparative evolutionary genomics unveils the molecular mechanism of reassignment of the CTG codon in Candida spp. Genome Res 2003, 13:544-557.

10. Kurtzman CP, Suzuki M: Phylogenetic analysis of ascomycete yeasts that form coenzyme Q-9 and the proposal of the new genera Babjeviella, Meyerozyma, Millerozyma, Priceomyces, and Scheffersomyces. Mycoscience 2010, 51:2-14.

11. Kurtzman CP: Phylogeny of the ascomycetous yeasts and the renaming of Pichia anomala to Wickerhamomyces anomalus. Antonie Van Leeuwenhoek 2011, 99:13-23.

12. Kurtzman C, Fell JW, Boekhout T: The Yeasts: A Taxonomic Study. 5th edition. London, Burlington, San Diego: Elsevier; 2011.

13. Kurtzman $C P$, Robnett $C J$ : Relationships among genera of the Saccharomycotina (Ascomycota) from multigene phylogenetic analysis of type species. FEMS Yeast Res 2013, 13:23-33.

14. Odronitz F, Kollmar M: Drawing the tree of eukaryotic life based on the analysis of 2,269 manually annotated myosins from 328 species. Genome Biol 2007, 8:R196.

15. Eckert C, Hammesfahr B, Kollmar M: A holistic phylogeny of the coronin gene family reveals an ancient origin of the tandem-coronin, defines a new subfamily, and predicts protein function. BMC Evol Biol 2011, 11:268.

16. Hammesfahr B, Kollmar M: Evolution of the eukaryotic dynactin complex, the activator of cytoplasmic dynein. BMC Evol Biol 2012, 12:95.

17. Butler G, Rasmussen MD, Lin MF, Santos MAS, Sakthikumar S, Munro CA, Rheinbay E, Grabherr M, Forche A, Reedy JL, Agrafioti I, Arnaud MB, Bates S, Brown AJP, Brunke S, Costanzo MC, Fitzpatrick DA, de Groot PWJ, Harris D, Hoyer LL, Hube B, Klis FM, Kodira C, Lennard N, Logue ME, Martin R, Neiman AM, Nikolaou E, Quail MA, Quinn J, et al: Evolution of pathogenicity and sexual reproduction in eight Candida genomes. Nature 2009, 459:657-662.

18. Ruby Programming Language. http://www.ruby-lang.org/.

19. Ruby on Rails. http://rubyonrails.org.

20. PyBioMaps is a framework to manage and visualize scientific data in a browser. http://pypi.python.org/pypi/PyBioMaps.

21. Altschul SF, Madden TL, Schäffer AA, Zhang J, Zhang Z, Miller W, Lipman DJ: Gapped BLAST and PSI-BLAST: A New Generation of Protein Database Search Programs. Nucleic Acids Res 1997, 25:3389-3402.

22. Camacho C, Coulouris G, Avagyan V, Ma N, Papadopoulos J, Bealer K, Madden TL: BLAST+: architecture and applications. BMC Bioinformatics 2009, 10:421.

23. Odronitz F, Kollmar M: Pfarao: a web application for protein family analysis customized for cytoskeletal and motor proteins (CyMoBase). BMC Genomics 2006, 7:300.

24. CyMoBase - a database for cytoskeletal and motor proteins. http://www cymobase.org

25. Stanke M, Waack S: Gene prediction with a hidden Markov model and a new intron submodel. Bioinformatics 2003, 19(2):ii215-ii225.

26. Keller O, Kollmar M, Stanke M, Waack S: A novel hybrid gene prediction method employing protein multiple sequence alignments. Bioinformatics 2011, 27(6):757-763.

27. Katoh $K$, Frith MC: Adding unaligned sequences into an existing alignment using MAFFT and LAST. Bioinformatics 2012, 28:3144-3146.

28. Needleman SB, Wunsch CD: A general method applicable to the search for similarities in the amino acid sequence of two proteins. $J \mathrm{Mol} B \mathrm{Bi} /$ 1970, 48:443-453.

29. Gotoh O: An improved algorithm for matching biological sequences. J Mol Biol 1982, 162:705-708.

30. Smith TF, Waterman MS: Identification of common molecular subsequences. Journal of Molecular Biology 1981, 147:195-197.

31. Hirschberg DS: A linear space algorithm for computing maximal common subsequences. Commun ACM 1975, 18:341-343.

32. Döring A, Weese $D$, Rausch $T$, Reinert $K$ : SeqAn An efficient, generic C++ library for sequence analysis. BMC Bioinformatics 2008, 9:11.

33. Talavera G, Castresana J: Improvement of phylogenies after removing divergent and ambiguously aligned blocks from protein sequence alignments. Syst Biol 2007, 56:564-577.

34. Price MN, Dehal PS, Arkin AP: FastTree 2-approximately maximum-likelihood trees for large alignments. PLOS ONE 2010, 5:e9490.

35. Lowe TM, Eddy SR: tRNAscan-SE: A Program for Improved Detection of Transfer RNA Genes in Genomic Sequence. Nucl Acids Res 1997, 25:0955-0964.

36. Odronitz F, Pillmann H, Keller O, Waack S, Kollmar M: WebScipio: an online tool for the determination of gene structures using protein sequences. BMC Genomics 2008, 9:422. 
37. Kollmar M, Lbik D, Enge S: Evolution of the eukaryotic ARP2/3 activators of the WASP family: WASP, WAVE, WASH, and WHAMM, and the proposed new family members WAWH and WAML. BMC Res Notes 2012, 5:88.

38. Keller O, Odronitz F, Stanke M, Kollmar M, Waack S: Scipio: using protein sequences to determine the precise exon/intron structures of genes and their orthologs in closely related species. BMC Bioinformatics 2008, 9:278.

39. Kent WJ: BLAT-the BLAST-like alignment tool. Genome Res 2002, 12:656-664.

40. Gabaldón T, Martin T, Marcet-Houben M, Durrens P, Bolotin-Fukuhara M, Lespinet O, Arnaise S, Boisnard S, Aguileta G, Atanasova R, Bouchier C, Couloux A, Creno S, Cruz JA, Devillers H, Enache-Angoulvant A, Guitard J, Jaouen L, Ma L, Marck C, Neuvéglise C, Pelletier E, Pinard A, Poulain J, Recoquillay J, Westhof E, Wincker P, Dujon B, Hennequin C, Fairhead C: Comparative genomics of emerging pathogens in the Candida glabrata clade. BMC Genomics 2013, 14:623.

41. Sugiyama H, Ohkuma M, Masuda Y, Park SM, Ohta A, Takagi M: In vivo evidence for non-universal usage of the codon CUG in Candida maltosa. Yeast 1995, 11:43-52

42. Tsui CKM, Daniel H-M, Robert V, Meyer W: Re-examining the phylogeny of clinically relevant Candida species and allied genera based on multigene analyses. FEMS Yeast Res 2008, 8:651-659.

43. Sekimoto S, Rochon D, Long JE, Dee JM, Berbee ML: A multigene phylogeny of Olpidium and its implications for early fungal evolution. BMC Evol Biol 2011, 11:331.

44. Verkley GJM, Quaedvlieg W, Shin H-D, Crous PW: A new approach to species delimitation in Septoria. Stud Mycol 2013, 75:213-305.

45. Hoffmann K, Pawłowska J, Walther G, Wrzosek M, de Hoog GS, Benny GL, Kirk PM, Voigt K: The family structure of the Mucorales: a synoptic revision based on comprehensive multigene-genealogies. Persoonia 2013, 30:57-76.

doi:10.1186/1471-2164-15-411

Cite this article as: Mühlhausen and Kollmar: Predicting the fungal CUG codon translation with Bagheera. BMC Genomics 2014 15:411.

\section{Submit your next manuscript to BioMed Central and take full advantage of:}

- Convenient online submission

- Thorough peer review

- No space constraints or color figure charges

- Immediate publication on acceptance

- Inclusion in PubMed, CAS, Scopus and Google Scholar

- Research which is freely available for redistribution 